\title{
On the new wave behavior of the Magneto-Electro-Elastic(MEE) circular rod longitudinal wave equation
}

\author{
Onur Alp İlhan ${ }^{*}$, Hasan Bulut ${ }^{b}$, Tukur A. Sulaiman $^{b}$ and Haci Mehmet Baskonus ${ }^{c}$ \\ ${ }^{a}$ Erciyes Univeristy, Faculty of Education, Melikgazi-Kayseri, Turkey \\ ${ }^{b}$ Firat University, Department of Mathematics, Elazig, Turkey \\ ${ }^{c}$ Harran University, Faculty of education, Department of Mathematics, Sanliurfa, Turkey \\ oailhan@erciyes.edu.tr, hbulut@firat.edu.tr,mtukur74@yahoo.com,hmbaskonus@gmail.com
}

\begin{tabular}{|c|c|}
\hline ARTICLE INFO & ABSTRACT \\
\hline $\begin{array}{l}\text { Article History: } \\
\text { Received } 15 \text { June } 2019 \\
\text { Accepted } 01 \text { September } 2019 \\
\text { Available } 04 \text { September } 2019\end{array}$ & $\begin{array}{l}\text { The analytical solution of the longitudinal wave equation in the MEE circular } \\
\text { rod is analyzed by the powerful sine-Gordon expansion method. Sine - Gordon } \\
\text { expansion is based on the well-known wave transformation and sine - Gordon } \\
\text { equation. In the longitudinal wave equation in mathematical physics, the trans- }\end{array}$ \\
\hline $\begin{array}{l}\text { Keywords: } \\
\text { The SGEM } \\
\text { Longitudinal wave equation } \\
\text { MEE circular rod }\end{array}$ & $\begin{array}{l}\text { verse Poisson MEE circular rod is caused by the dispersion. Some solutions } \\
\text { with complex, hyperbolic and trigonometric functions have been successfully } \\
\text { implemented. Numerical simulations of all solutions are given by selecting the } \\
\text { appropriate parameter values. The physical meaning of the analytical solution }\end{array}$ \\
\hline
\end{tabular}

AMS Classification 2010:

$35 L 05 ; 58 J 45$

\section{Introduction}

Innovative analytical new solutions for non-linear evolution equations (NEEs) has very important role in area of non-linear physics. Non-linear evolution equations are often used to state complex models that appear in different areas of non-linear science, such as biological sciences, quantum mechanics, and plasma physics. Recently, different analytical techniques have been invested to search new types of solutions. NLEs such as the new general algebra method [1], the $\tan \left(\frac{F(\xi)}{2}\right)$ expansion method [2], the extended tanh method [3], the jacobi elliptic function method [4, the homogeneous balance method [5], the generalized Kudryashov method [6], the generalized $\left(G^{\prime} / G\right)$ method [7, the extended homoclinic test function method [8], the improved Bernoulli sub-equation function method [9], the improved $\exp (-\Phi(\xi))$ expansion function method [10] and so on. In general, many more analytical techniques have been designed and used in obtaining analytical solutions of different NLEs [1] 22]. Authors of
23 28 obtained new lump and interaction for some of models in which arise in applied sciences. Moreover, Manafian and co-authors [29,30] used the analytical methods for getting to exact solutions.

The powerful sine-Gordon expansion method (SGEM) [31, 32 was used to find some new solution methods to the longitudinal wave equation of the magneto-electro-elastic (MEE) circular rod [33 in this study. The longitudinal wave equation of the MEE circular rod is developed by [33], the longitudinal wave equation is a dispersion equation caused by the transverse Poisson's effect in MEE circular rod, developed from [34];

$$
u_{t t}-q^{2} u_{x x}-\left(\frac{q}{2} u^{2}+p u_{t t}\right)_{x x}=0
$$

where $p$ is the dispersion parameter and $q$ is the linear longitudinal wave velocity of the MEE circular rod which depend on material properties and rod geometry 34. Different analytical methods have been put in place to find solutions

*Corresponding Author 
to the longitudinal wave equation in magnetoelectro-elastic MEE circular rod, like the improved $\left(G^{\prime} / G\right)$-expansion method [35], the functional variable method [36], the ansatz method [37, etc.

\section{The SGEM}

The general cases of SGEM was given in this section,

Take into account the following sine-Gordon equation [38], [39]:

$$
u_{x x}-u_{t t}=n^{2} \sin (u)
$$

where $u=u(x, t)$ and $n \in \mathbb{R} \backslash\{0\}$.

Using the wave transformation $u=u(x, t)=$ $U(\beta), \beta=\alpha(x-k t)$ on Eq. (2), following nonlinear ordinary differential equation (NODE) was gotten as:

$$
U^{\prime \prime}=\frac{n^{2}}{\alpha^{2}\left(1-k^{2}\right)} \sin (U),
$$

as $U=U(\beta)$, the amplitude of the traveling wave is $\beta$ and $k$ is the speed of the traveling wave. To integrate the equation (3), we get the following equation:

$$
\left[\left(\frac{U}{2}\right)^{\prime}\right]^{2}=\frac{n^{2}}{\alpha^{2}\left(1-k^{2}\right)} \sin ^{2}\left(\frac{U}{2}\right)+Q
$$

as the integral constant is $Q$.

Set $Q=0, \phi(\beta)=\frac{U}{2}$ and $b^{2}=\frac{n^{2}}{\alpha^{2}\left(1-k^{2}\right)}$ in Eq. (41), gives:

$$
\phi^{\prime}=b \sin (\phi),
$$

inserting $b=1$ into Eq. (5), produces:

$$
\phi^{\prime}=\sin (\phi),
$$

simplifying Eq. (6), creates the following two important equations;

$$
\begin{aligned}
& \sin (\phi)=\sin (\phi(\beta))=\left.\frac{2 d e^{\beta}}{d^{2} e^{2 \beta}+1}\right|_{d=1}=\operatorname{sech}(\beta), \\
& \cos (\phi)=\cos (\phi(\beta))=\left.\frac{d^{2} e^{2 \beta}-1}{d^{2} e^{2 \beta}+1}\right|_{d=1}=\tanh (\beta),
\end{aligned}
$$

as the integral constant is $d$.

For the given non-linear partial differential equation Eq. (9);

$$
P\left(u, u u_{x}, u^{2} u_{t}, \ldots\right),
$$

its solution in the form as;

$U(\beta)=\sum_{i=1}^{m} \tanh ^{i-1}(\beta)\left[B_{i} \operatorname{sech}(\beta)+A_{i} \tanh (\beta)\right]+A_{0}$.

Equation (10) may be given according to Eq. (17) and (8) as;

$U(\phi)=\sum_{i=1}^{m} \cos ^{i-1}(\phi)\left[B_{i} \sin (\phi)+A_{i} \cos (\phi)\right]+A_{0}$.

$m$ is determined by balancing the highest power non-linear term and the highest derivative in the transformed NODE. Taking each summation of the coefficients of $\sin ^{i}(w) \cos ^{j}(w), 0 \leq i, j \leq m$ to be zero, produces a set of equations. This set of equation is solved with the symbolic computational computational software, yields the values of the coefficients $A_{i}, B_{i}, \mu$ and $c$. Eventually, inserting the produced values of these coefficients into Eq. (10) accompanied by the value of $m$, gives the fresh travelling wave solutions to Eq. (91).

\section{Applications}

The SGEM is used in searching the fresh solutions to Eq. (1) in this section. Considering Eq. (1), the following NODE was gotten by using the wave transformation; $u=U(\beta), \beta=\mu(-k t+x)$;

$$
2 p k^{2} \mu^{2} U^{\prime \prime}-2\left(k^{2}-c_{0}^{2}\right) U+c_{0}^{2} U^{2}=0,
$$

$p$ is non-zero constant and we get $m=2$ by balancing $U^{\prime \prime}$ and $U^{2}$ in Eq. (12).

Using Eq. (11) together with the value $m=2$, we get the following equation;

$$
\begin{aligned}
U(\phi)= & B_{1} \sin (\phi)+A_{1} \cos (\phi)+B_{2} \cos (\phi) \sin (\phi) \\
& +A_{2} \cos ^{2}(\phi)+A_{0},
\end{aligned}
$$

differentiating Eq. (13) twice, we get: 


$$
\begin{aligned}
U^{\prime \prime}(\phi) & =B_{1} \cos ^{2}(\phi) \sin (\phi)-B_{1} \sin ^{3}(\phi) \\
& -2 A_{1} \sin ^{2}(\phi) \cos (\phi)+B_{2} \cos ^{3}(\phi) \sin (\phi) \\
& -5 B_{2} \sin ^{3}(\phi) \cos (\phi)-4 A_{2} \cos ^{2}(\phi) \sin ^{2}(\phi) \\
& +2 A_{2} \sin ^{4}(\phi),
\end{aligned}
$$

Setting Eq. (13) and (15) to Eq. (12), generating trigonometric equations. After replacing the trigonometric constants in the trigonometric equation, a set of algebraic equations is collected by setting each sum of the coefficients of the trigonometric functions of the same power to zero. The set of equations is solved with assistance of symbolic mathematical softwares; to get coefficient values for different cases. We insert coefficient values for each case into the Eq. (10) with a value of $m=2$, this gives us a new solution Eq. (1).

\section{Case-1:}

$A_{0}=4\left(1+\frac{k^{2}}{q^{2}}\right), A_{1}=0, B_{1}=0, A_{2}=-6\left(1-\frac{k^{2}}{q^{2}}\right)$,

$B_{2}=-6 i+\frac{6 i k^{2}}{q^{2}}, p=\frac{1}{k^{2} \mu^{2}}\left(k^{2}-q^{2}\right)$.

\section{Case-2:}

$$
\begin{aligned}
A_{0} & =4-\frac{4}{1+p \mu^{2}}, A_{1}=0, B_{1}=0 \\
A_{2} & =-6+\frac{6}{1+p \mu^{2}}, B_{2}=\frac{6 p \mu^{2}\left(p \mu^{2}-1\right)}{p^{2} \mu^{4}-1} i \\
q & =-k \sqrt{1+p \mu^{2}} .
\end{aligned}
$$

\section{Case-3:}

$$
\begin{aligned}
& A_{0}=-6+\frac{6 k^{2}}{q^{2}}, A_{1}=0, B_{1}=0, A_{2}=6-\frac{6 k^{2}}{q^{2}}, \\
& B_{2}=6 i\left(1-\frac{k^{2}}{q^{2}}\right), \mu=-\frac{1}{k \sqrt{p}} \sqrt{\left(k^{2}-q^{2}\right)} .
\end{aligned}
$$

\section{Case-4:}

$A_{0}=1+\frac{k^{2}}{q^{2}}, A_{1}=0, B_{1}=0, A_{2}=-3\left(1-\frac{k^{2}}{q^{2}}\right)$,

$B_{2}=0, p=\frac{k^{2}-q^{2}}{4 k^{2} \mu^{2}}$.

\section{Case-5:}

$$
\begin{aligned}
& A_{0}=1-\frac{1}{4 p \mu^{2}+1}, A_{1}=0, B_{1}=0 \\
& A_{2}=-3+\frac{3}{4 p \mu^{2}+1}, B_{2}=0, q=k \sqrt{4 p \mu^{2}+1}
\end{aligned}
$$

\section{Case-6:}

$$
\begin{aligned}
& A_{0}=1-\frac{k^{2}}{q^{2}}, A_{1}=0, B_{1}=0, A_{2}=3\left(\frac{k^{2}}{q^{2}}-1\right), \\
& B_{2}=0, \mu=\frac{1}{2 k \sqrt{p}} \sqrt{\left(k^{2}-q^{2}\right)} i .
\end{aligned}
$$

\section{Solutions:}

(1). The following solution is gotten by with case 1 ;

$$
\begin{aligned}
u_{1}(x, t) & =\frac{6\left(k^{2}-q^{2}\right)}{q^{2}}(1+i \operatorname{sech}[\mu(x-k t)] \\
& \left.\times \tanh [\mu(x-k t)]-\tanh [(-k t+x) \mu]^{2}\right)
\end{aligned}
$$
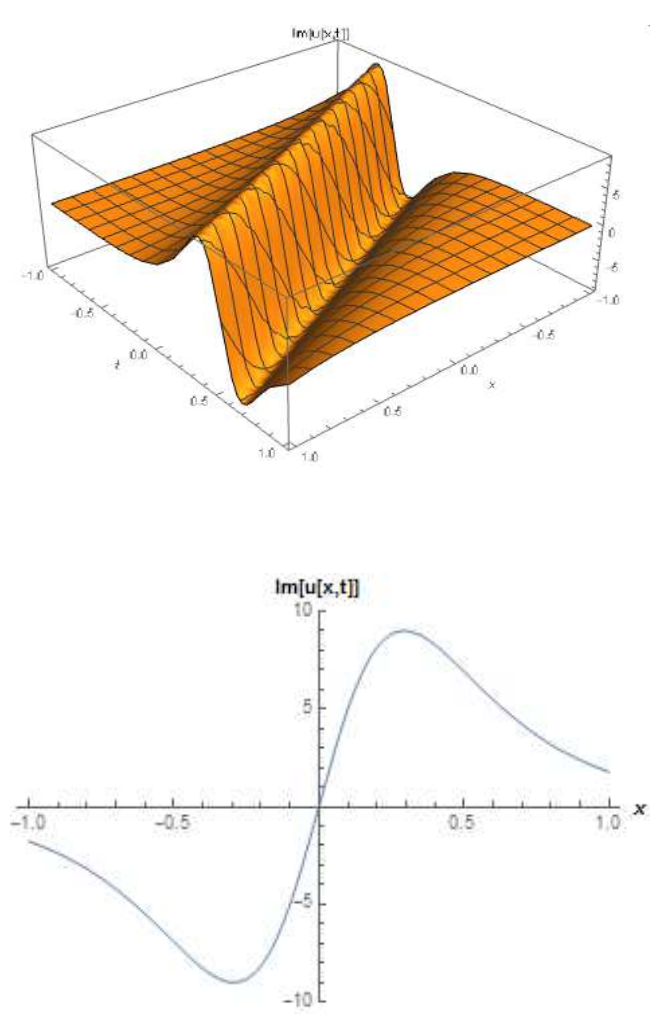

Figure 1. The 3D shape for the imaginary part of Eq. (15) with the values $k=2, c_{0}=1, \mu=3,-3<$ $x<3,-5<t<5$ and $t=0$ for the graphic of $2 \mathrm{D}$.

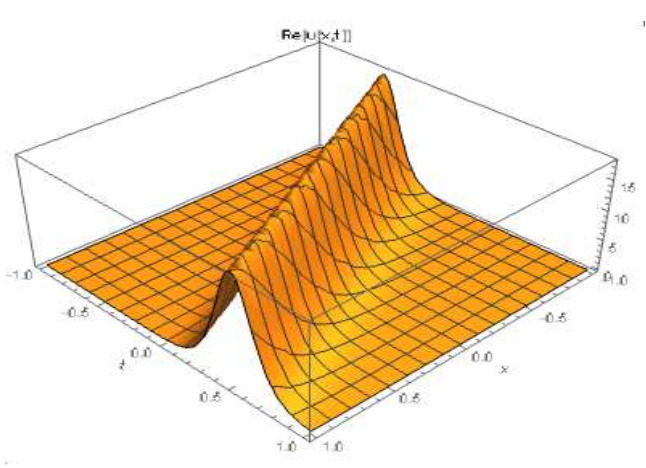




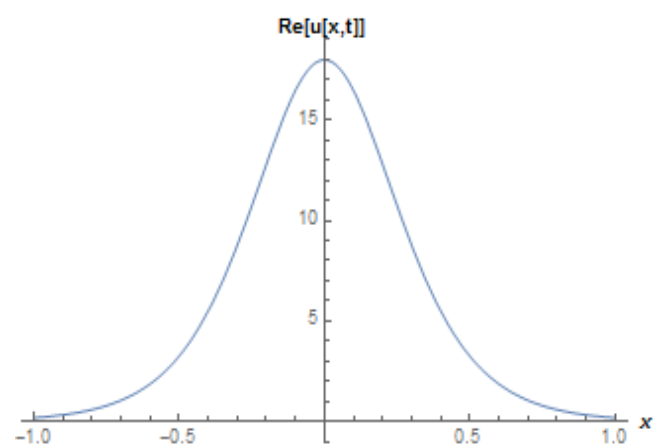

Figure 2. The 3D shape for the real part of Eq. (15) with the values $k=2, c_{0}=1, \mu=3,-3<x<3$, $-5<t<5$ and $t=0$ for the graphic of $2 \mathrm{D}$.

(2). The following solution is gotten by with case 2 ;

$$
\begin{aligned}
u_{2}(x, t) & =4-\frac{4}{1+p \mu^{2}}+\left(6 i p \mu^{2}\left(-1+p \mu^{2}\right)\right) . \\
& . \frac{\sec h[(-k t+x) \mu] \tanh [-k t+x) \mu]}{-1+p^{2} \mu^{4}} \\
& +\left(-6+\frac{6}{1+p^{2} \mu^{2}}\right) \tanh [(-k t+x) \mu]^{2} .
\end{aligned}
$$
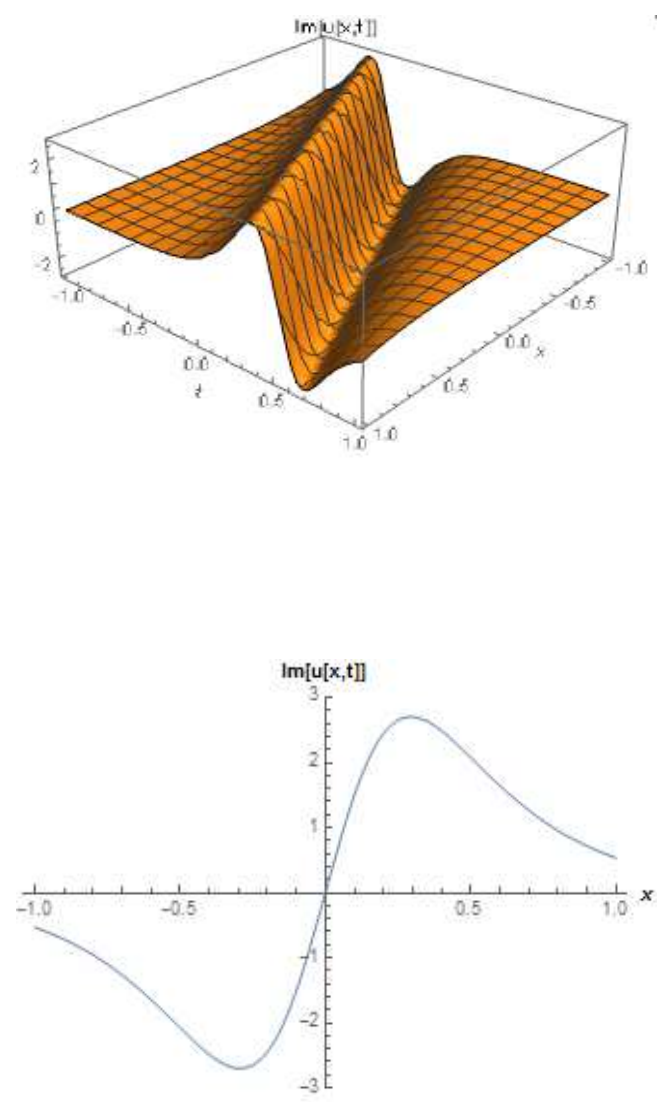
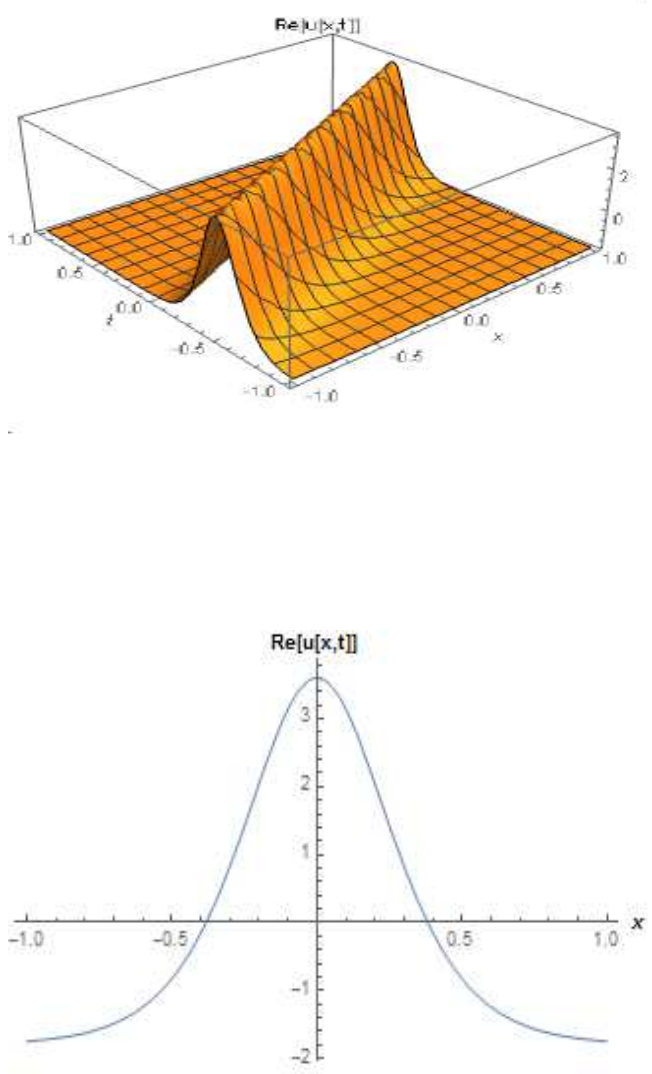

Figure 3. The 2D and 3D shape for the imaginary and real part of Eq. (16) with the values $k=2, p=1$, $\mu=3,-5<x<8,0<t<2$ and $t=0$ for the graphics of $2 \mathrm{D}$.

(3). The following solution is gotten by with case 3 ;

$$
\begin{aligned}
u_{3}(x, t) & =\frac{6}{q^{2}}\left(q^{2}-k^{2}\right)\left(-1-i \sec h\left[\frac{1}{k \sqrt{p}}\left(\sqrt{k^{2}-q^{2}}\right)\right.\right. \\
& \times(x-k t)] \tanh \left[\frac{1}{k \sqrt{p}}\left(\sqrt{k^{2}-q^{2}}\right)(x-k t)\right]+ \\
& \left.+\tanh \left[\frac{1}{k \sqrt{p}}\left(\sqrt{k^{2}-q^{2}}\right)(x-k t)\right]^{2}\right) .
\end{aligned}
$$



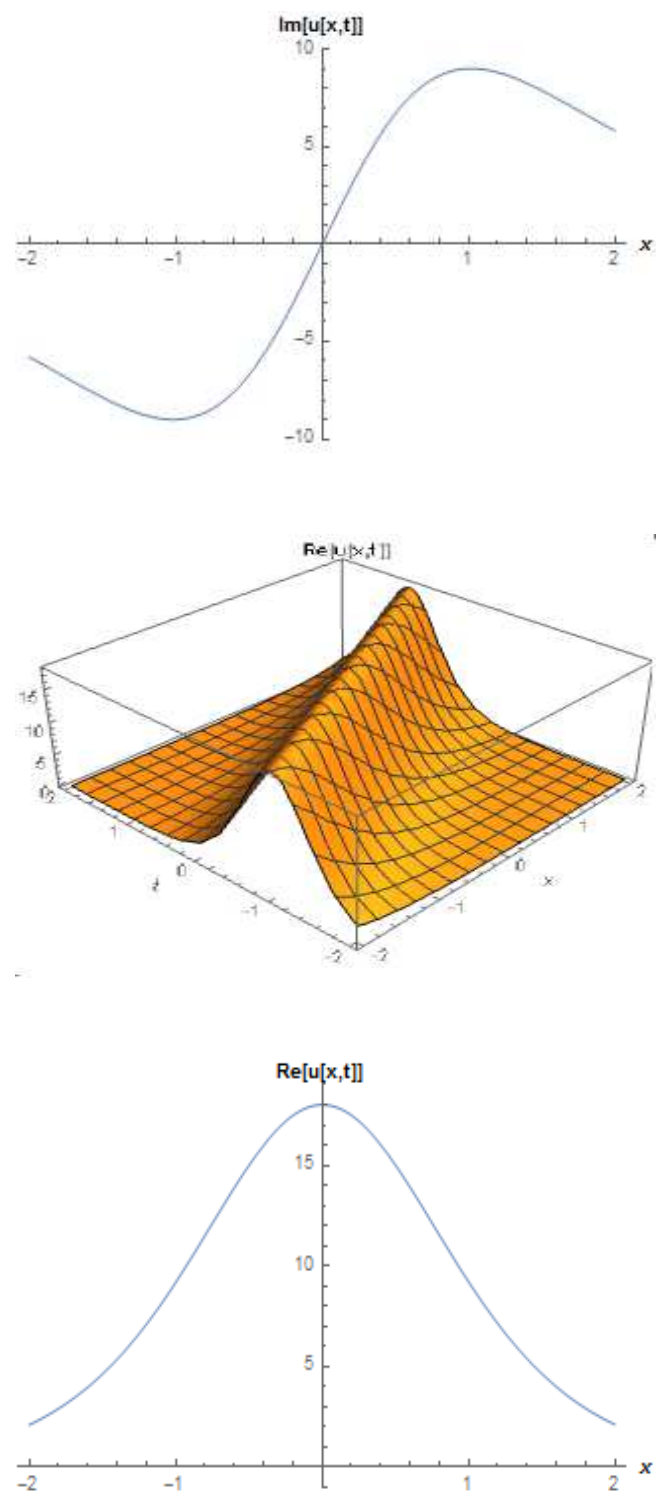

Figure 4. The $2 \mathrm{D}$ and $3 \mathrm{D}$ shape for the imaginary and real part of Eq. (17) with the values $k=2, p=1$, $c_{0}=1,-5<x<5,0<t<2$ and $t=0$ for the graphics of $2 \mathrm{D}$.

(4). The following solution is gotten by with case 4 ;

$$
u_{4}(x, t)=\frac{k^{2}-q^{2}}{q^{2}}\left(2-3 \tanh [(-k t+x) \mu]^{2}\right)
$$

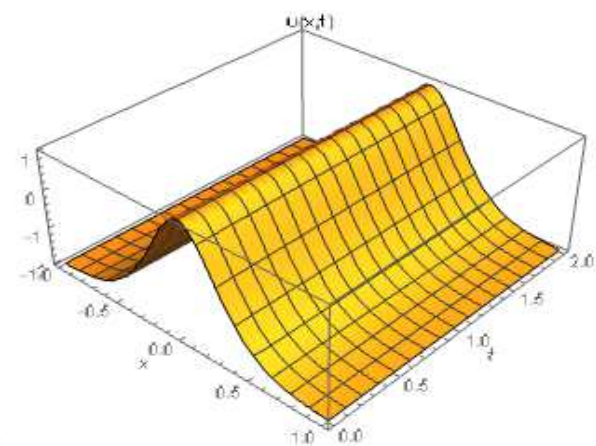

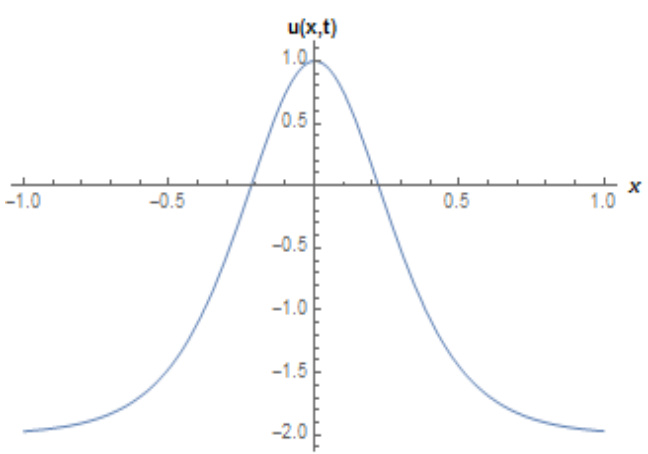

Figure 5. The 2D and 3D shape for the Eq. (18) with the values $k=$ $0.005, \mu=3, c_{0}=1,-1<x<1$, $0<t<2$ and $t=0$ for the graphic of $2 \mathrm{D}$.

(5). The following solution is gotten by with case 5

$$
u_{5}(x, t)=\frac{4 p \mu^{2}}{1+4 p \mu^{2}}\left(1-3 \tanh [\mu(x-k t)]^{2}\right)
$$
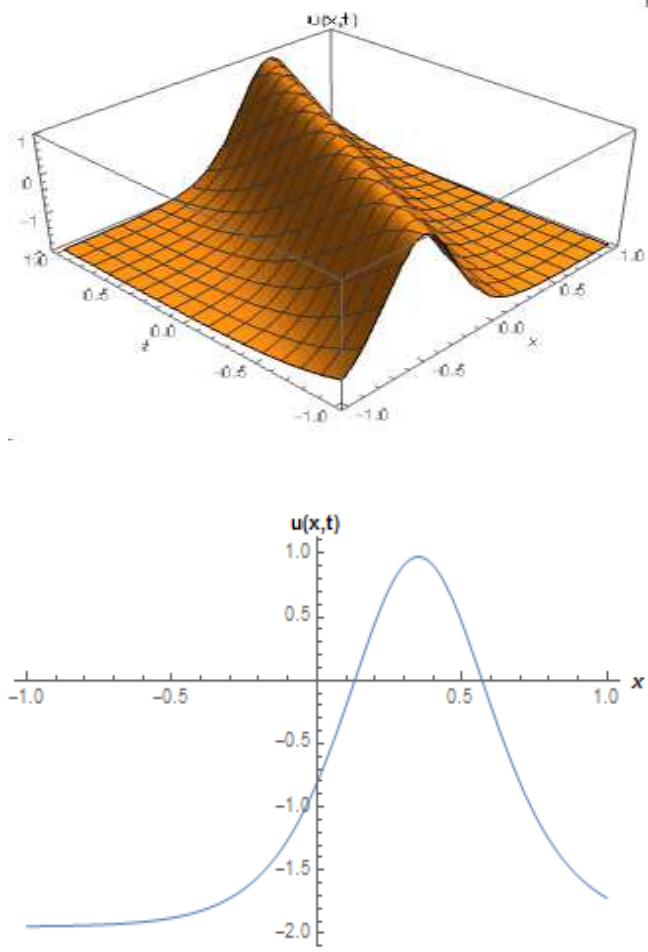

Figure 6. The 2D and 3D shape for the Eq. (19) with the values $k=0.5$, $\mu=3, p=1,-0.5<x<1,0<t<2$ and $t=0.7$ for the graphic of $2 \mathrm{D}$.

(6). The following solution is gotten by with case 6 ;

$$
u_{6}(x, t)=\frac{k^{2}-q^{2}}{q^{2}}\left(-1-3 \tan \left[\frac{\sqrt{k^{2}-q^{2}}}{2 k \sqrt{p}}(x-k t)\right]^{2}\right) .
$$



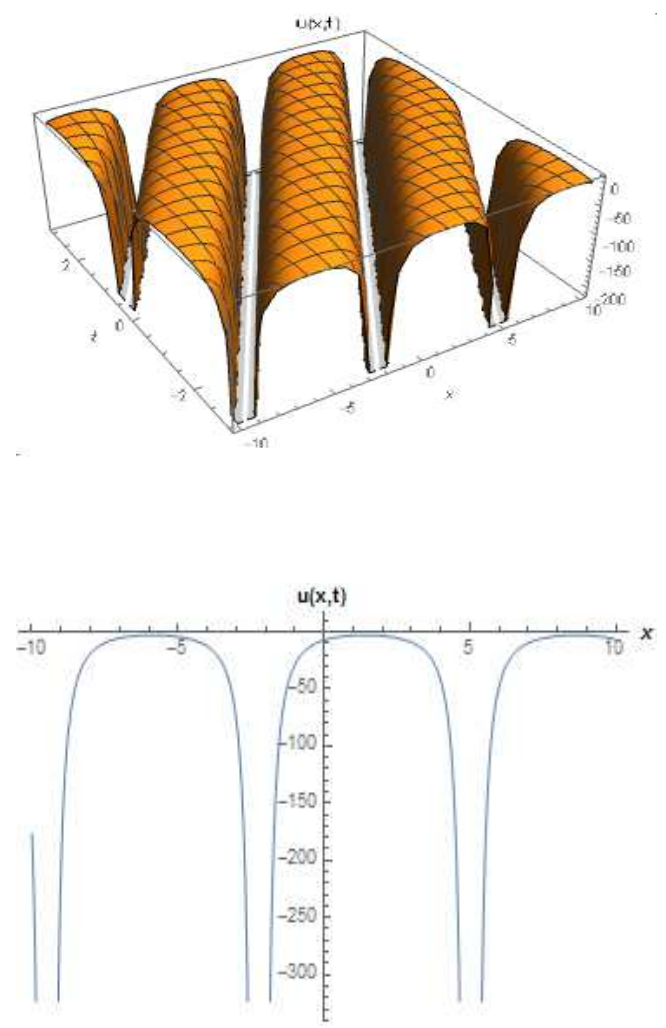

Figure 7. The 2D and 3D shape for the Eq. (20) with the values $k=2$, $c_{0}=1, p=1,-0.5<x<1$, $0<t<2$ and $t=0.7$ for the graphic of $2 \mathrm{D}$.

\section{Results and Discussion}

In [33] the improved $\exp (-\Phi(\xi))$-expansion function method is used in the solution of the magneto-electro-elastic circular rod longitudinal wave equation and the solution of different hyperbolic function forms is obtained. Secondly, the well-known improvement $\left(G^{\prime} / G\right)$-expansion method [35] has been used for this equation and some precise hyperbolic and trigonometric functions are obtained. We observe that our results are new, but have the same solution structure. When compared with the existing, the results obtained by using these two methods. On the other hand, we observe that in the numerical simulations of the solutions we presented; Figure 1, Figure 2 and Figure 7 are singular soliton surfaces, Figure 3 is solit off surface, Figures 4-6 are soliton surfaces. We observe that some solutions in this study have important physical significance, such as the emergence of hyperbolic tangents in the calculation of magnetic moments and relative velocities, the emergence of hyperbolic secant in the profile of a laminar jet 40 .

\section{Conclusions}

In this study, by utilizing the sine-Gordon extension method with the help of symbolic mathematical software, we investigated the solution of the magneto-electro-elastic circular rod longitudinal wave equation. We obtain some new solutions for complex hyperbolic and trigonometric functions. All solutions obtained in this study validate wave equations in magneto-electro-elastic circular rod and we examine this using the same procedure as symbolic mathematical software. We performed numerical simulations of all the solutions obtained in this paper. We observed that our results may be helpful in detecting transverse Poissons effect magneto-electro-elastic circular rod. The SineGordon extension method is a powerful and efficient mathematical tool that can be used with the help of symbolic mathematical software to explore different non-linear methods arising in different fields of non-linear science.

\section{Acknowledgments}

The authors gratefully thank the referees for their several suggestions and comments.

\section{References}

[1] Ren, Y.J., Liu S.T., and Zhang, H.Q. (2007). A new generalized algebra method and its application in the $(2+1)$-dimensional Boiti-Leon-Pempinelli equation. Chaos Solitons and Fractals, 32, 1655-1665.

[2] Manafian, J. (2016). Optical soliton solutions for Schrödinger type nonlinear evolution equations by the $\tan (F(\xi) / 2)$-expansion method. Optik-International Journal of Light and Electron Optics, 127, 4222-4245.

[3] Feng, W.G., Li, K.M., Li, Y.Z. and Lin, C. (2009). Explicit exact solutions for $(2+1)$ dimensional Boiti-Leon-Pempinelli equation. Communications in Non-linear Science and Numerical Simulation, 14, 2013-2017.

[4] Fu, Z., Liu, S. and Zhao, Q. (2001) New Jacobi elliptic function expansion and new periodic solutions of nonlinear wave equations. Physics Letters A, 290, 72-76.

[5] Zayed, E.M.E. and Alurrfi, K.A.E. (2014). The homogeneous balance method and its applications for finding the exact solutions for nonlinear evolution equations. Italian Journal of Pure and Applied Mathematics, 33, 307318.

[6] Islam, M.S, Khan, K. and Arnous, A.H. (2015). Generalized Kudryashov method for solving some $(3+1)$-dimensional nonlinear 
evolution equations. New Trends in Mathematical Sciences, 3(3), 46-57.

[7] Lu, H.L., Liu, X.Q. and Niu, L. (2010). A generalized $\left(G^{\prime} / G\right)$-expansion method and its applications to nonlinear evolution equations. Applied Mathematics and Computation, 215, 3811-3816.

[8] Wang, C. (2016). Dynamic behavior of traveling waves for the Sharma-Tasso-Olver equation. Non-linear Dynamics, 85(2), 1119-1126.

[9] Baskonus, H.M. and Bulut, H. (2016). Exponential prototype structures for $(2+1)$ - dimensional Boiti-Leon-Pempinelli systems in mathematical physics. Waves in Random and Complex Media, 26(2), 201-208.

[10] Baskonus, H.M., Bulut, H. and Belgacem, F.B.M. (2017). Analytical Solutions for nonlinear long-short wave interaction systems with highly complex structure. Journal of Computational and Applied Mathematics, 312, 257-266.

[11] Kadkhoda, N. and Jafari, H. (2016). Kudryashov method for exact solutions of isothermal magnetostatic atmospheres. Applied Mathematics and Computation, 6(1), 4352.

[12] El-wakil, S.A., El-labany, S.K., Zahran, M.A. and Sabry, R. (2002). Modified extended tanh-function method for solving nonlinear partial differential equations. Physics Letters A, 299, 179-188.

[13] Islam, M.S., Khan, K., Arnous, A.H. (2015). Generalized Kudryashov method for solving some (3+1)-dimensional nonlinear evolution equations. New Trends in Mathematical Sciences, 3(3), 46-57.

[14] Chen, H.T., Hong-Qing, Z. (2004). New double periodic and multiple soliton solutions of the generalized $(2+1)$-dimensional Boussinesq equation. Chaos Solitons and Fractals, 20, 765-769.

[15] Baskonus, H.M. and Bulut, H. (2015). On some new analytical solutions for The $(2+1)$ dimensional burgers equation and the special type of Dodd- Bullough-Mikhailov equation. Journal of Applied Analysis and Computation, 5(4), 613-625.

[16] Lu, Z. and Zhang, H. (2004). Soliton like and multi-soliton like solutions for the BoitiLeon-Pempinelli equation. Chaos, Solitons and Fractals, 19, 527-531.

[17] Huang, D.J. and Zhang, H.Q. (2004). Exact Travelling Wave Solutions for the BoitiLeon-Pempinelli Equation. Chaos Solitons and Fractals, 22, 243-247.
[18] Dai, C. and Wang, Y. (2009). Periodic structures based on variable separation solution of the $(2+1)$-dimensional Boiti-Leon-Pempinelli equation. Chaos Solitons and Fractals, 39, 350-355.

[19] Liang, Y. (2014). Exact solutions of the (3+1)-dimensional modified KdV- ZakharovKuznetsev equation and Fisher equations using the modified simple equation method. Journal of Interdisciplinary Mathematics, 17, 565-578.

[20] Zhang, H. (2007). Extended jacobi elliptic function expansion method and its applications. Communications in Non-linear Science and Numerical Simulation, 12(5), 627-635.

[21] Petrovic, N.Z. and Bohra, M. (2016). General jacobi elliptic function expansion method applied to the generalized $(3+1)$-dimensional nonlinear Schrodinger equation. Optical and Quantum Electronics, 48, (268).

[22] Yan, Z. (2003). Jacobi elliptic function solutions of nonlinear wave equations via the new sinh-gordon equation expansion method. $M M$ Research Preprints, 22, 363-375.

[23] Manafian, J. and Lakestani, M. (2019). Lump-type solutions and interaction phenomenon to the bidirectional Sawada- Kotera equation. Pramana, 92:41.

[24] Manafian, J. (2018). Novel solitary wave solutions for the $(3+1)$-dimensional extended Jimbo-Miwa equations. Computers $\& 5$ Mathematics with Applications, 76(5), 1246-1260.

[25] Manafian, J., Mohammadi-Ivatloo, B. and Abapour, M. (2019). Lump-type solutions and interaction phenomenon to the $(2+1)$ dimensional breaking soliton equation. $A p$ plied Mathematics and Computation Volume, $356,13-41$.

[26] Ilhan, O.A., Manafian, J. and Shahriari M. (2019). Lump wave solutions and the interaction phenomenon for a variable coefficient Kadomtsev-Petviashvili equation. Computers \& Mathematics with Applications, In press.

[27] Foroutan, M., Manafian, J. and Ranjbaran, A. (2018). Lump solution and its interaction to $(3+1)$-D potential-YTSF equation. Nonlinear Dynamics, 92, 2077-2092.

[28] Mohammad, S. and Manafian, J. (2019). Analytical behaviour of lump solution and interaction phenomenon to the KadomtsevPetviashvili-like equation. Pramana, 93:3.

[29] Seadawy, A.R. and Manafian, J. (2018). New soliton solution to the longitudinal wave equation in a magneto-electro-elastic circular rod. Results in Physics, 8, 1158-1167. 
[30] Ramin, M.T., Manafian, J., Baskonus, H.M. and Duşunceli, F. (2019). Applications of He's semi-inverse variational method and ITEM to the nonlinear long-short wave interaction system, IJAAS Journal, 6(8), 53-64.

[31] Yan, C. (1996). A simple transformation for nonlinear waves. Physics Letters A , 22(4), 77-84.

[32] Bulut, H., Sulaiman, T.A. and Baskonus, H.M. (2016). New solitary and optical wave structures to the Korteweg-de Vries equation with dual-power law nonlinearity. Optical and Quantum Electronics, 48:564, 1-14.

[33] Baskonus, H.M., Bulut, H. and Atangana, A. (2016). On the complex and hyperbolic structures of longitudinal wave equation in a Magneto-Electro-Elastic circular rod. Smart Materials and Structures, 25(3), 035022.

[34] Xue, C.X., Pan, E. and Zhang, X.Y. (2011). Solitary waves in a Magneto-Electro-Elastic circular rod. Smart Materials and Structures, 20(10), 035022.

[35] Ma, X., Pan, Y. and Chang, L. (2013). Explicit travelling wave solutions in a MagnetoElectro-Elastic circular rod. International Journal of Computer Science Issues, 10(1), 62-68.

[36] Khan, K., Koppelaar, H. and Akbar, A. (2016). Exact and numerical soliton solutions to nonlinear wave equations. Computational and Mathematical Engineering, 2, 5-22.

[37] Younis, M. and Ali, S. (2015). Dark and singular solitons in Magneto-Electro-Elastic circular rod. Waves in Random and Complex Media, 25(4), 549-555.

[38] Yan, Z. and Zhang, H. (1999). New explicit and exact travelling wave solutions for a system of variant Boussinesq equations in mathematical physics. Physics Letters A, 252, 291296

[39] Zhen-Ya, Y., Hong-oing, Z. and En-Gui, F. (1999). New explicit and travelling wave solutions for a class of nonlinear evolution equations. Acta Physica Sinica, 48(1), 1-5.
[40] Weisstein, E.W. (2002). Concise encyclopedia of nathematics. 2nd edition (New York: CRC Press).

Onur Alp Ilhan received his Masters Degree (2002) in Mathematics from Erciyes University and obtained a PhD Degree (2005) from National University of Uzbekistan. Mr. lhan is currently working as an Associate Professor at Faculty of Education in Erciyes University,. His research interests include Mathbio., ODE, PDE and Integral equations.

(10) http://orcid.org/0000-0003-1618-6439

Hasan Bulut is currently professor of Mathematics in Firat University. His research interests include stochastic differential equations, fluid and heat mechanics, finite element method, analytical methods for nonlinear differential equations and numerical solutions of the partial differential equations.

(10) http://orcid.org/0000-0002-6089-1517

Tukur A. Sulaiman is a research assistant at Firat University, Turkey and an assistant lecturer as Federal University Dutse, Nigeria. He is currently pursuing his PhD. (Applied Mathematics) in Firat University, Turkey. He has so far published 4 articles in various journals. His research interests include; stochastic optimization, analytical and numerical solutions of nonlinear ordinary/partial differential equations including the fractional differential equations.

(10)http://orcid.org/0000-0001-7284-8332

Haci Mehmet Baskonus received the PhD degree in Mathematics from the Firat University, Turkey, in 2014. He is currently an Assoc. Prof. Dr at Faculty of Education in Harran University. His research interests include ordinary and partial differential equations, analytical methods for linear and nonlinear differential equations, mathematical physics, numerical solutions of the partial differential equations, fractional differential equations (of course ordinary and partial) and computer programming like Mathematica. (1) http://orcid.org/0000-0003-4085-3625

An International Journal of Optimization and Control: Theories \& Applications (http://ijocta.balikesir.edu.tr)

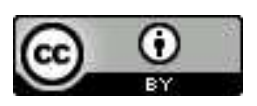

This work is licensed under a Creative Commons Attribution 4.0 International License. The authors retain ownership of the copyright for their article, but they allow anyone to download, reuse, reprint, modify, distribute, and/or copy articles in IJOCTA, so long as the original authors and source are credited. To see the complete license contents, please visit http://creativecommons.org/licenses/by/4.0/. 